\title{
Future Higgs Studies: A Theorist's Outlook
}

\author{
Howard E. Haber* \\ Santa Cruz Institute for Particle Physics \\ University of California, Santa Cruz, CA 95064 USA \\ E-mail: haberescipp.ucsc.edu
}

\begin{abstract}
We examine some of the theoretical and phenomenological implications of the Higgs boson discovery and discuss what these imply for future Higgs studies at the LHC and future colliders. In particular, one of the outstanding unanswered questions is whether additional scalars beyond the observed Higgs boson are present in the spectrum of fundamental particles. Any theory of a non-minimal Higgs sector must possess a scalar state whose properties are approximately those of the Standard Model Higgs boson. This can be achieved in the so-called alignment limit of the extended scalar sector. Examples of scalar sectors in which an approximate alignment limit is achieved are surveyed.
\end{abstract}

Prospects for Charged Higgs Discovery at Colliders

3-6 October 2016

Uppsala, Sweden

${ }^{*}$ Speaker. 


\section{Introduction}

The discovery of the Higgs boson in 2012 [1,2] marked an important milestone in the study of fundamental particles and their interactions. The Standard Model (SM) of particle physics is now complete. Indeed, there are no definitive departures from the Standard Model observed in experiments conducted at high energy collider facilities. Nevertheless, some fundamental microscopic phenomena must necessarily lie outside of the purview of the SM. These include: neutrinos with non-zero mass [3]; dark matter [4]; the baryon asymmetry of the universe [5]; the suppression of CP-violation in the strong interactions (the so-called strong CP problem [6]); gauge coupling unification [7]; inflation in the early universe [8]; dark energy [9]; and the gravitational interaction. None of these phenomena can be explained within the framework of the SM alone.

As a result, the SM should be regarded at best as a low-energy effective field theory, which is valid below some high energy scale $\Lambda$. For example, a credible theory of neutrino masses (e.g., the type-I seesaw model [3]) posits the existence of a right-handed electroweak singlet Majorana neutrino of mass of order $10^{14} \mathrm{GeV}$. The gravitational force is governed by Planck-scale physics corresponding to $\Lambda \sim 10^{19} \mathrm{GeV}$. Henceforth, we shall define $\Lambda$ to be the lowest energy scale at which the SM breaks down. The predictions made by the SM depend on a number of parameters that must be taken as input to the theory. These parameters are sensitive to ultraviolet (UV) physics, and since the physics at very high energies is not known, one cannot predict their values. In general, fermions and boson masses depend differently on $\Lambda$ [10]. On the one hand, fermion masses are logarithmically sensitive to UV physics, due to the chiral symmetry of massless fermions, i.e. $\delta m_{F} \sim m_{f} \ln \left(\Lambda^{2} / m_{F}^{2}\right)$. In contrast, no such symmetry exists to protect masses of spin-0 bosons (in the absence of supersymmetry), and consequently we expect quadratic sensitivity of the scalar boson squared mass to UV physics, $\delta m_{B}^{2} \sim \Lambda^{2}$.

In the SM, the Higgs scalar potential,

$$
V(\Phi)=-\mu^{2}\left(\Phi^{\dagger} \Phi\right)+\frac{1}{2} \lambda\left(\Phi^{\dagger} \Phi\right)^{2}
$$

where $\mu^{2}=\frac{1}{2} \lambda v^{2}$ depends on the vacuum expectation value (vev) $v$ of the Higgs field. The parameter $\mu^{2}$ is quadratically sensitive to $\Lambda$. Hence, to obtain $v \simeq 246 \mathrm{GeV}$ in a theory where $v \ll \Lambda$ requires a significant fine-tuning of the ultraviolet parameters of the fundamental theory. Indeed, the one-loop contribution to the squared mass parameter $\mu^{2}$ would be expected to be of order $\left(g^{2} / 16 \pi^{2}\right) \Lambda^{2}$. Setting this quantity to be of order of $v^{2}$ (to avoid an unnatural cancellation between the tree-level parameter and the loop corrections) yields $\Lambda \simeq 4 \pi v / g \sim O(1 \mathrm{TeV})$. A natural theory of electroweak symmetry breaking (EWSB) would seem to require new physics at the $\mathrm{TeV}$ scale associated with the EWSB dynamics.

There have been a number of theoretical proposals to explain the origin of the EWSB energy scale: (1) naturalness is restored by supersymmetry which ties the bosons to the more well-behaved fermions [11]; (2) the Higgs boson is an approximate Goldstone boson, the only other known mechanism for keeping an elementary scalar light [12]; (3) The Higgs boson is a composite scalar, with an inverse length of order the TeV-scale [12]; (4) the EWSB scale is chosen by some vacuum selection mechanism [13]. Of course, maybe none of these explanations are relevant, and the EWSB energy scale (which appears to us to be highly fine-tuned) is simply the result of some initial condition whose origin will never be discernible. 
In light of these remarks, how do we make further progress? We have at our disposal a very successful experimental particle physics facility-the Large Hadron Collider (LHC), which has only begun a comprehensive probe of the TeV-energy scale. To the experimentalists, I say: "keep searching for new physics beyond the SM (BSM)." Any observed departures from SM predictions will contain critical clues to a more fundamental theory of elementary particles and their interactions. To the theorists, I say: "find new examples of BSM physics (which might provide a natural explanation to the EWSB scale) that may have been overlooked in LHC searches." But what if no signals for BSM physics emerge soon? My answer is: "look to the Higgs sector." After all, we have only recently discovered a most remarkable particle that seems to be like nothing that has ever been seen before-an elementary scalar boson. Shouldn't we probe this state thoroughly and explore its properties with as much precision as possible?

Putting considerations of naturalness aside, two critical questions to be addressed in future LHC experimentation are:

1. Are there additional Higgs bosons to be discovered? (This includes new charged scalars of interest to this conference.) If fermionic matter and the gauge sector of the SM are non-minimal, why shouldn't scalar matter also be non-minimal? To paraphrase I.I. Rabi, "who ordered that?"

2. If we measure the Higgs properties with sufficient precision, will deviations from SM-like Higgs behavior be revealed?

One might be concerned that adding additional Higgs scalars to the theory will exacerbate the fine-tuning problem associated with the EWSB scale. Of course, there are many examples in which natural explanations of the EWSB scale employ BSM physics with extended Higgs sectors. The minimal supersymmetric extension of the SM (MSSM), which employs two Higgs doublets, is the most well known example of this type, but there are numerous other BSM examples as well. If you give up on naturalness (e.g., vacuum selection), it has been argued that it may be difficult to accommodate more than one Higgs doublet at the electroweak scale [14]. However, it is possible to construct "partially natural" extended Higgs sectors in which one scalar squared mass parameter is fine-tuned (as in the SM), but additional scalar mass parameters are related to the EWSB scale by a symmetry [15].

In the rest of this talk, I will take an agnostic approach and entertain the possibility of an extended Higgs sector without providing a specific theoretical motivation. I shall focus on the theoretical constraints on extended Higgs sectors in light of current experimental data (including the fact that the observed Higgs boson is SM-like). These constraints will provide an important framework for considering the phenomenology of additional Higgs bosons that could be discovered in future experimentation at the LHC (or at future colliders currently under consideration).

\section{Theoretical implications of a SM-like Higgs boson}

Based on the Run-I LHC Higgs data [16], it is already apparent that the observed Higgs boson is SM-like. Thus any model of BSM physics, including models of extended Higgs sectors, must incorporate this observation. In models of extended Higgs sectors, a SM-like Higgs boson can be achieved in a particular limit of the model called the alignment limit [17-21].

Consider an extended Higgs sector with $n$ hypercharge-one Higgs doublets $\Phi_{i}$ and $m$ additional singlet Higgs fields $\phi_{i}$. After minimizing the scalar potential, we assume that only the neutral scalar 
fields acquire vevs (in order to preserve electromagnetic charge conservation), $\left\langle\Phi_{i}^{0}\right\rangle=v_{i} / \sqrt{2}$ and $\left\langle\phi_{j}^{0}\right\rangle=x_{j}$, where $v^{2} \equiv \sum_{i}\left|v_{i}\right|^{2}=4 m_{W}^{2} / g^{2} \simeq(246 \mathrm{GeV})^{2}$. We define new linear combinations, $H_{i}$, of the hypercharge-one doublet Higgs fields (this is the so-called Higgs basis [22-24]). In particular,

$$
H_{1}=\left(\begin{array}{c}
H_{1}^{+} \\
H_{1}^{0}
\end{array}\right)=\frac{1}{v} \sum_{i} v_{i}^{*} \Phi_{i}, \quad\left\langle H_{1}^{0}\right\rangle=v / \sqrt{2},
$$

and $H_{2}, H_{3}, \ldots, H_{n}$ are the other mutually orthogonal linear combinations of doublet scalar fields such that $\left\langle H_{i}^{0}\right\rangle=0$ (for $i \neq 1$ ). That is $H_{1}^{0}$ is aligned in field space with the direction of the scalar field vev. In the alignment limit, $h \equiv \sqrt{2} \operatorname{Re} H_{1}^{0}-v$ is a mass-eigenstate, and the tree-level couplings of $h$ to itself, to gauge bosons and to fermions are precisely those of the SM Higgs boson. In general, $\sqrt{2} \operatorname{Re} H_{1}^{0}-v$ is not a mass-eigenstate due to mixing with other neutral scalars. Thus, the observed Higgs boson is SM-like if at least one of the following two conditions are satisfied:

1. The diagonal squared masses of the other scalar fields are all large compared to the mass of the observed Higgs boson (the so-called decoupling limit [17,25]), and/or

2. The elements of the scalar squared mass matrix that govern the mixing of $\sqrt{2} \operatorname{Re} H_{1}^{0}-v$ with other neutral scalars are suppressed.

In the $\mathrm{SM}, m_{h}^{2}=\lambda v^{2}$ where $v \simeq 246 \mathrm{GeV}$, and $\lambda$ is the Higgs self-coupling [cf. eq. (1.1)] which should not be much larger than $O(1)$. Thus, we expect $m_{h} \sim O(v)$. In extended Higgs sectors, there can be a new mass parameter, $M \gg v$, such that all physical Higgs masses with one exception are of $O(M)$. The Higgs boson, with $m_{h} \sim O(v)$, is SM-like due to approximate alignment. This is the decoupling limit. After integrating out all the heavy degrees of freedom at the mass scale $M$, one is left with a low-energy effective theory which consists of the SM particles, including a single neutral scalar boson. This low-energy effective theory is precisely the SM!

The alignment limit is most naturally achieved in the decoupling regime. However, in this case the additional Higgs boson states are very heavy and may be difficult to observe at the LHC. In the case of approximate alignment without decoupling ${ }^{1}$ (due to suppressed scalar mixing), non-SMlike Higgs boson states need not be very heavy and thus are more easily accessible at the LHC.

\section{Examples of extended Higgs sectors near the alignment limit}

\subsection{Extending the SM Higgs sector with a singlet scalar}

The simplest example of an extended Higgs sector adds a real scalar field $S$. The most general renormalizable gauge-invariant scalar potential (subject to a $\mathbb{Z}_{2}$ symmetry to eliminate linear and cubic terms in $S$ ) is [29-31]

$$
V(\Phi, S)=-m^{2} \Phi^{\dagger} \Phi-\mu^{2} S^{2}+\frac{1}{2} \lambda_{1}\left(\Phi^{\dagger} \Phi\right)^{2}+\frac{1}{2} \lambda_{2} S^{4}+\lambda_{3}\left(\Phi^{\dagger} \Phi\right) S^{2} .
$$

After minimizing the scalar potential, $\left\langle\Phi^{0}\right\rangle=v / \sqrt{2}$ and $\langle S\rangle=x / \sqrt{2}$. The squared mass matrix of the neutral Higgs bosons is $[32,33]$

$$
\mathscr{M}^{2}=\left(\begin{array}{ll}
\lambda_{1} v^{2} & \lambda_{3} v x \\
\lambda_{3} v x & \lambda_{2} x^{2}
\end{array}\right)
$$

\footnotetext{
${ }^{1}$ In some models, alignment without decoupling can be achieved by a symmetry $[26,27]$. The inert doublet model [28] is a noteworthy example in which the exact alignment limit is a consequence of a discrete $\mathbb{Z}_{2}$ symmetry. In most cases, approximate alignment without decoupling is an accidental region of the model parameter space.
} 
The corresponding mass eigenstates are $h$ and $H$ with $m_{h} \leq m_{H}$. As discussed in Section 2, an approximate alignment limit can be realized in two different ways: either $\left|\lambda_{3}\right| x \ll v$ and/or $x \gg v$. In the case where $\left|\lambda_{3}\right| x \ll v, h$ is SM-like if $\lambda_{1} v^{2}<\lambda_{2} x^{2}$ and $H$ is SM-like if $\lambda_{1} v^{2}>\lambda_{2} x^{2}$. In contrast, $x \gg v$ corresponds to the decoupling limit, where $h$ is SM-like and $m_{H} \gg m_{h}$.

The Higgs mass eigenstates are explicitly defined via

$$
\left(\begin{array}{l}
h \\
H
\end{array}\right)=\left(\begin{array}{rr}
\cos \alpha & -\sin \alpha \\
\sin \alpha & \cos \alpha
\end{array}\right)\left(\begin{array}{c}
\sqrt{2} \operatorname{Re} \Phi^{0}-v \\
\sqrt{2} S-x
\end{array}\right)
$$

where

$$
\begin{aligned}
& \lambda_{1} v^{2}=m_{h}^{2} \cos ^{2} \alpha+m_{H}^{2} \sin ^{2} \alpha, \\
& \lambda_{2} x^{2}=m_{h}^{2} \sin ^{2} \alpha+m_{H}^{2} \cos ^{2} \alpha, \\
& \lambda_{3} x v=\left(m_{H}^{2}-m_{h}^{2}\right) \sin \alpha \cos \alpha .
\end{aligned}
$$

The SM-like Higgs boson is approximately given by $\sqrt{2} \operatorname{Re} \Phi^{0}-v$.

If $h$ is SM-like, then $m_{h}^{2} \simeq \lambda_{1} v^{2}$ and

$$
|\sin \alpha|=\frac{\left|\lambda_{3}\right| v x}{\sqrt{\left(m_{H}^{2}-m_{h}^{2}\right)\left(m_{H}^{2}-\lambda_{1} v^{2}\right)}} \simeq \frac{\left|\lambda_{3}\right| v x}{m_{H}^{2}-m_{h}^{2}} \ll 1,
$$

If $H$ is SM-like, then $m_{H}^{2} \simeq \lambda_{1} v^{2}$ and

$$
|\cos \alpha|=\frac{\left|\lambda_{3}\right| v x}{\sqrt{\left(m_{H}^{2}-m_{h}^{2}\right)\left(\lambda_{1} v^{2}-m_{h}^{2}\right)}} \simeq \frac{\left|\lambda_{3}\right| v x}{m_{H}^{2}-m_{h}^{2}} \ll 1 .
$$

A phenomenological analysis presented in Ref. [33] shows that the allowed parameter regime (consistent with the LHC Higgs data) roughly satisfies $|\sin \alpha| \lesssim 0.3$ if $m_{H} \gtrsim m_{h}=125 \mathrm{GeV}$ and $|\sin \alpha| \gtrsim 0.9$ if $m_{h}<m_{H}=125 \mathrm{GeV}$.

\subsection{The two-Higgs doublet model (2HDM)}

Consider the 2HDM with hypercharge-one, doublet fields $\Phi_{1}$ and $\Phi_{2}[34,35]$. After minimizing the scalar potential, $\left\langle\Phi_{i}^{0}\right\rangle=v_{i} / \sqrt{2}$ (for $i=1,2$ ), where $v_{1}^{2}+v_{2}^{2} \simeq(246 \mathrm{GeV})^{2}$ and $\tan \beta \equiv v_{2} / v_{1}$. The Higgs basis fields are defined as,

$$
H_{1}=\left(\begin{array}{c}
H_{1}^{+} \\
H_{1}^{0}
\end{array}\right) \equiv \frac{v_{1}^{*} \Phi_{1}+v_{2}^{*} \Phi_{2}}{v}, \quad H_{2}=\left(\begin{array}{c}
H_{2}^{+} \\
H_{2}^{0}
\end{array}\right) \equiv \frac{-v_{2} \Phi_{1}+v_{1} \Phi_{2}}{v},
$$

such that $\left\langle H_{1}^{0}\right\rangle=v / \sqrt{2}$ and $\left\langle H_{2}^{0}\right\rangle=0$. The Higgs basis is uniquely defined up to an overall rephasing of the Higgs basis field $\mathrm{H}_{2}$.

In the Higgs basis, the scalar potential is given by [23, 24,36]:

$$
\begin{gathered}
V=Y_{1} H_{1}^{\dagger} H_{1}+Y_{2} H_{2}^{\dagger} H_{2}+\left[Y_{3} H_{1}^{\dagger} H_{2}+\text { h.c. }\right]+\frac{1}{2} Z_{1}\left(H_{1}^{\dagger} H_{1}\right)^{2}+\frac{1}{2} Z_{2}\left(H_{2}^{\dagger} H_{2}\right)^{2}+Z_{3}\left(H_{1}^{\dagger} H_{1}\right)\left(H_{2}^{\dagger} H_{2}\right) \\
+Z_{4}\left(H_{1}^{\dagger} H_{2}\right)\left(H_{2}^{\dagger} H_{1}\right)+\left\{\frac{1}{2} Z_{5}\left(H_{1}^{\dagger} H_{2}\right)^{2}+\left[Z_{6}\left(H_{1}^{\dagger} H_{1}\right)+Z_{7}\left(H_{2}^{\dagger} H_{2}\right)\right] H_{1}^{\dagger} H_{2}+\text { h.c. }\right\},
\end{gathered}
$$

where $Y_{1}, Y_{2}$ and $Z_{1}, \ldots, Z_{4}$ are real, whereas $Y_{3}, Z_{5}, Z_{6}$ and $Z_{7}$ are potentially complex. After minimizing the scalar potential, $Y_{1}=-\frac{1}{2} Z_{1} v^{2}$ and $Y_{3}=-\frac{1}{2} Z_{6} v^{2}$. 
For simplicity, we consider here the case of a CP-conserving scalar potential. ${ }^{2}$ In this case, one can rephase the Higgs basis field $H_{2}$ such that $\operatorname{Im} Z_{5}=\operatorname{Im} Z_{6}=\operatorname{Im} Z_{7}=0$. We identify the CP-odd Higgs boson as $A=\sqrt{2} \operatorname{Im} H_{2}^{0}$, with $m_{A}^{2}=Y_{2}+\frac{1}{2}\left(Z_{3}+Z_{4}-Z_{5}\right) v^{2}$. After eliminating $Y_{2}$ in favor of $m_{A}^{2}$, the CP-even Higgs squared mass matrix with respect to the Higgs basis states, $\left\{\sqrt{2} \operatorname{Re} H_{1}^{0}-v, \sqrt{2} \operatorname{Re} H_{2}^{0}\right\}$, is given by

$$
\mathscr{M}_{H}^{2}=\left(\begin{array}{cc}
Z_{1} v^{2} & Z_{6} v^{2} \\
Z_{6} v^{2} & m_{A}^{2}+Z_{5} v^{2}
\end{array}\right) .
$$

The CP-even Higgs bosons are $h$ and $H$ with $m_{h} \leq m_{H}$. The couplings of $\sqrt{2} \operatorname{Re} H_{1}^{0}-v$ coincide with those of the SM Higgs boson. ${ }^{3}$ Thus, the alignment limit corresponds to two limiting cases:

1. $\left|Z_{6}\right| \ll 1$. In this case, $h$ is SM-like if $m_{A}^{2}+\left(Z_{5}-Z_{1}\right) v^{2}>0$; otherwise, $H$ is SM-like.

2. $m_{A}^{2} \gg\left(Z_{1}-Z_{5}\right) v^{2}$. This is the decoupling limit; $h$ is SM-like and $m_{A} \sim m_{H} \sim m_{H^{ \pm}} \gg m_{h}$. In particular, the $\mathrm{CP}$-even mass eigenstates are:

$$
\left(\begin{array}{c}
H \\
h
\end{array}\right)=\left(\begin{array}{rr}
c_{\beta-\alpha} & -s_{\beta-\alpha} \\
s_{\beta-\alpha} & c_{\beta-\alpha}
\end{array}\right)\left(\begin{array}{c}
\sqrt{2} \operatorname{Re} H_{1}^{0}-v \\
\sqrt{2} \operatorname{Re} H_{2}^{0}
\end{array}\right),
$$

where $c_{\beta-\alpha} \equiv \cos (\beta-\alpha)$ and $s_{\beta-\alpha} \equiv \sin (\beta-\alpha)$ are defined in terms of the angle $\alpha$ that diagonalizes the CP-even Higgs squared mass matrix when expressed in the original basis of scalar fields, $\left\{\sqrt{2} \operatorname{Re} \Phi_{1}^{0}-v_{1}, \sqrt{2} \operatorname{Re} \Phi_{2}^{0}-v_{2}\right\}$, and $\tan \beta \equiv v_{2} / v_{1}$. Since the SM-like Higgs boson is approximately $\sqrt{2} \operatorname{Re} H_{1}^{0}-v$, it follows that $h$ is SM-like if $\left|c_{\beta-\alpha}\right| \ll 1$, and $H$ is SM-like if $\left|s_{\beta-\alpha}\right| \ll 1$.

The approximate alignment limit can be derived more explicitly as follows. The CP-even Higgs squared mass matrix yields [38,39]

$$
\begin{aligned}
& Z_{1} v^{2}=m_{h}^{2} s_{\beta-\alpha}^{2}+m_{H}^{2} c_{\beta-\alpha}^{2}, \\
& Z_{6} v^{2}=\left(m_{h}^{2}-m_{H}^{2}\right) s_{\beta-\alpha} c_{\beta-\alpha}, \\
& Z_{5} v^{2}=m_{H}^{2} s_{\beta-\alpha}^{2}+m_{h}^{2} c_{\beta-\alpha}^{2}-m_{A}^{2} .
\end{aligned}
$$

If $h$ is SM-like, then $m_{h}^{2} \simeq Z_{1} v^{2}$ and

$$
\left|c_{\beta-\alpha}\right|=\frac{\left|Z_{6}\right| v^{2}}{\sqrt{\left(m_{H}^{2}-m_{h}^{2}\right)\left(m_{H}^{2}-Z_{1} v^{2}\right)}} \simeq \frac{\left|Z_{6}\right| v^{2}}{m_{H}^{2}-m_{h}^{2}} \ll 1 .
$$

The decoupling limit is realized when $m_{H} \gg m_{h}$. In contrast, alignment without decoupling requires that $\left|Z_{6}\right| \ll 1$ and $m_{H} \sim O(v)$. If $H$ is SM-like, then $m_{H}^{2} \simeq Z_{1} v^{2}$ and [40]

$$
\left|s_{\beta-\alpha}\right|=\frac{\left|Z_{6}\right| v^{2}}{\sqrt{\left(m_{H}^{2}-m_{h}^{2}\right)\left(Z_{1} v^{2}-m_{h}^{2}\right)}} \simeq \frac{\left|Z_{6}\right| v^{2}}{m_{H}^{2}-m_{h}^{2}} \ll 1,
$$

which can only be achieved if $\left|Z_{6}\right| \ll 1$. In particular a SM-like $H$ can only arise in the limit of alignment without decoupling.

\footnotetext{
${ }^{2}$ The more general case in which no scalar basis exists such that all the parameters of eq. (3.10) are simultaneously real is treated in Refs. [36,37].

${ }^{3}$ Although the tree-level couplings of $\sqrt{2} \operatorname{Re} H_{1}^{0}-v$ coincide with those of the SM Higgs boson, the one-loop couplings can differ due to the exchange of non-minimal Higgs states (if not too heavy). For example, the charged Higgs boson loop interferes with the $W$ and fermion loop contributions to the amplitude for the decay of the SM-like Higgs boson to $\gamma \gamma$ or $\gamma Z$.
} 
So far, we have not yet discussed the couplings of the Higgs bosons to fermions. In the $\Phi_{1}-\Phi_{2}$ basis, the 2HDM Higgs-quark Yukawa Lagrangian is [37]:

$$
-\mathscr{L}_{\mathrm{Y}}=\bar{U}_{L} \Phi_{i}^{0 *} h_{i}^{U} U_{R}-\bar{D}_{L} K^{\dagger} \Phi_{i}^{-} h_{i}^{U} U_{R}+\bar{U}_{L} K \Phi_{i}^{+} h_{i}^{D \dagger} D_{R}+\bar{D}_{L} \Phi_{i}^{0} h_{i}^{D \dagger} D_{R}+\text { h.c. }
$$

where $K$ is the CKM mixing matrix, $h^{U, D}$ are $3 \times 3$ Yukawa coupling matrices, and there is an implicit sum over $i=1,2$. Unlike in the SM, the diagonalization of the quark masses does not automatically diagonalize the neutral-Higgs-quark Yukawa coupling matrices. Hence, the general 2HDM possesses tree-level Higgs-mediated flavor-changing neutral currents (FCNCs) which are generically too large and thus inconsistent with experimental data. In order to naturally eliminate tree-level Higgs-mediated FCNC [41,42], one can impose a discrete symmetry to restrict the structure of $\mathscr{L}_{\mathrm{Y}}$. Two different choices for how the discrete symmetry acts on the quarks then yield: Type-I Yukawa couplings [43,44] if $h_{1}^{U}=h_{1}^{D}=0$, and Type-II Yukawa couplings $[44,45]$ if $h_{1}^{U}=h_{2}^{D}=0$. (Similar considerations can also be applied to the Higgs-lepton Yukawa couplings.)

It is straightforward to work out the Higgs couplings in the approximate alignment limit. Some examples are provided in Table 1 in the case where $h$ is SM-like with a CP-conserving scalar potential and Type-I or II Yukawa couplings. In the third column of Table 1, the first non-trivial corrections to the alignment limit are presented. Note that these corrections are correlated, and the approach to decoupling is governed by $c_{\beta-\alpha}$. Thus, any deviations from SM-like behavior of the observed Higgs boson can provide important clues to the structure of the extended Higgs sector. The phenomenology of the $2 \mathrm{HDM}$ in the approximate alignment limit and its implications for future LHC experimental studies have recently been elucidated in Refs. $[39,40]$ in the case where $h$ or $H$, respectively, is identified as the observed Higgs boson of mass $125 \mathrm{GeV}$.

\begin{tabular}{|c||c|c|}
\hline Higgs interaction & 2HDM coupling & approach to alignment limit \\
\hline$h V V$ & $s_{\beta-\alpha}$ & $1-\frac{1}{2} c_{\beta-\alpha}^{2}$ \\
$h h h$ & $*$ & $1+2\left(Z_{6} / Z_{1}\right) c_{\beta-\alpha}$ \\
$h H^{+} H^{-}$ & $*$ & $\frac{1}{3}\left[\left(Z_{3} / Z_{1}\right)+\left(Z_{7} / Z_{1}\right) c_{\beta-\alpha}\right]$ \\
$H h h$ & $*$ & $-Z_{6} / Z_{1}+\left[1-\frac{2}{3}\left(Z_{345} / Z_{1}\right)\right] c_{\beta-\alpha}$ \\
$h h h h$ & $*$ & $1+3\left(Z_{6} / Z_{1}\right) c_{\beta-\alpha}$ \\
$h \bar{D} D$ & $s_{\beta-\alpha} \mathbb{1}+c_{\beta-\alpha} \rho_{R}^{D}$ & $\mathbb{1}+c_{\beta-\alpha} \rho_{R}^{D}$ \\
$h \bar{U} U$ & $s_{\beta-\alpha} \mathbb{1}+c_{\beta-\alpha} \rho_{R}^{U}$ & $\mathbb{1}+c_{\beta-\alpha} \rho_{R}^{U}$ \\
\hline
\end{tabular}

Table 1: The 2HDM couplings of the SM-like Higgs boson $h$ normalized to those of the SM Higgs boson, in the approach to the alignment limit. The $h H^{+} H^{-}$and $H h h$ couplings are normalized to the SM $h h h$ coupling [17,39] (where $Z_{345} \equiv Z_{3}+Z_{4}+Z_{5}$ ). The scalar Higgs potential is taken to be CP-conserving. For the Higgs couplings to fermions, $D$ is a column vector of three down-type fermion fields (either down-type quarks or charged leptons) and $U$ is a column vector of three up-type quark fields. For Type-I Yukawa couplings, $\rho_{R}^{D}=\rho_{R}^{U}=\mathbb{1} \cot \beta$, and for Type-II Yukawa couplings, $\rho_{R}^{D}=-\mathbb{1} \tan \beta$ and $\rho_{R}^{U}=\mathbb{1} \cot \beta$ [19]. In the third column above, the first non-trivial correction to alignment is exhibited. Finally, complete expressions for the entries marked with a $*$ can be found in Refs. [36,37]. 


\subsection{The MSSM Higgs sector}

The MSSM Higgs sector is a CP-conserving Type-II 2HDM. The dimension-four terms of the scalar potential are constrained by supersymmetry. In particular, the scalar potential parameters (at tree level) in the Higgs basis are determined by the electroweak gauge couplings [36],

$$
\begin{array}{ll}
Z_{1}=Z_{2}=\frac{1}{4}\left(g^{2}+g^{\prime 2}\right) c_{2 \beta}^{2}, & Z_{5}=\frac{1}{4}\left(g^{2}+g^{\prime 2}\right) s_{2 \beta}^{2}, \quad Z_{7}=-Z_{6}=\frac{1}{4}\left(g^{2}+g^{\prime 2}\right) s_{2 \beta} c_{2 \beta}, \\
Z_{3}=Z_{5}+\frac{1}{4}\left(g^{2}-g^{\prime 2}\right), & Z_{4}=Z_{5}-\frac{1}{2} g^{2},
\end{array}
$$

in a convention where $\tan \beta \geq 0$, where $c_{2 \beta} \equiv \cos 2 \beta$ and $s_{2 \beta} \equiv \sin 2 \beta$. It then follows from eq. (3.13) that,

$$
\cos ^{2}(\beta-\alpha)=\frac{m_{Z}^{4} s_{2 \beta}^{2} c_{2 \beta}^{2}}{\left(m_{H}^{2}-m_{h}^{2}\right)\left(m_{H}^{2}-m_{Z}^{2} c_{2 \beta}^{2}\right)} .
$$

The decoupling limit is achieved when $m_{H} \gg m_{h}$ as expected. Exact alignment without decoupling is (naively) possible at tree-level when $Z_{6}=0$, which yields $\sin 4 \beta=0$ and $m_{h}^{2}=Z_{1} v^{2}=m_{Z}^{2} c_{2 \beta}^{2}$. However, these results are inconsistent with the observed Higgs mass of $125 \mathrm{GeV}$.

It is well known that radiative corrections can significantly modify the tree-level Higgs properties [46]. In particular, consider the limit where $m_{h}, m_{A}, m_{H}, m_{H^{ \pm}} \ll M_{S}$, where $M_{S}^{2} \equiv m_{\tilde{t}_{1}} m_{\tilde{t}_{2}}$ is the product of top squark masses. In this case, one can formally integrate out the squarks and generate a low-energy effective 2HDM Lagrangian (which is no longer of the tree-level MSSM form). At one-loop, the dominant contributions to the effective $Z_{1}$ and $Z_{6}$ parameters are given by [47] ${ }^{4}$

$$
\begin{aligned}
& Z_{1} v^{2}=m_{Z}^{2} c_{2 \beta}^{2}+\frac{3 v^{2} s_{\beta}^{4} h_{t}^{4}}{8 \pi^{2}}\left[\ln \left(\frac{M_{S}^{2}}{m_{t}^{2}}\right)+\frac{X_{t}^{2}}{M_{S}^{2}}\left(1-\frac{X_{t}^{2}}{12 M_{S}^{2}}\right)\right], \\
& Z_{6} v^{2}=-s_{2 \beta}\left\{m_{Z}^{2} c_{2 \beta}-\frac{3 v^{2} s_{\beta}^{2} h_{t}^{4}}{16 \pi^{2}}\left[\ln \left(\frac{M_{S}^{2}}{m_{t}^{2}}\right)+\frac{X_{t}\left(X_{t}+Y_{t}\right)}{2 M_{S}^{2}}-\frac{X_{t}^{3} Y_{t}}{12 M_{S}^{4}}\right]\right\},
\end{aligned}
$$

where $s_{\beta} \equiv \sin \beta, h_{t}$ is the top quark Yukawa coupling, $X_{t} \equiv A_{t}-\mu \cot \beta$ and $Y_{t} \equiv A_{t}+\mu \tan \beta$.

Note that $m_{h}^{2} \simeq Z_{1} v^{2}$ is consistent with $m_{h} \simeq 125 \mathrm{GeV}$ for suitable choices for $M_{S}$ and $X_{t}$. Exact alignment (i.e., $Z_{6}=0$ ) can now be achieved due to an accidental cancellation between tree-level and loop contributions [47],

$$
m_{Z}^{2} c_{2 \beta}=\frac{3 v^{2} s_{\beta}^{2} h_{t}^{4}}{16 \pi^{2}}\left[\ln \left(\frac{M_{S}^{2}}{m_{t}^{2}}\right)+\frac{X_{t}\left(X_{t}+Y_{t}\right)}{2 M_{S}^{2}}-\frac{X_{t}^{3} Y_{t}}{12 M_{S}^{4}}\right] .
$$

One can manipulate eq. (3.20) into a 7th order polynomial equation in $\tan \beta$. The alignment condition is then achieved by (numerically) solving this equation for positive real solutions of $\tan \beta$. Following a recipe provided by Refs. [48,49], one can further improve eqs. (3.18) and (3.19) to include the leading two-loop corrections of $O\left(\alpha_{S} h_{t}^{2}\right)$ by replacing $h_{t}$ with $h_{t}(\lambda)$, where $\lambda \equiv\left[m_{t}\left(m_{t}\right) M_{S}\right]^{1 / 2}$ in the one-loop leading log contributions and $\lambda \equiv M_{S}$ in the leading threshold corrections. Imposing $Z_{6}=0$ now leads to a 11 th order polynomial equation in $\tan \beta$ that can be solved numerically. Three positive solutions are exhibited in Fig. 1 as a function of $\mu / M_{S}$ and $A_{t} / M_{S}$ [50].

\footnotetext{
${ }^{4} \mathrm{CP}$-violating phases, which could appear in the MSSM parameters such as $\mu$ and $A_{t}$, are neglected.
} 

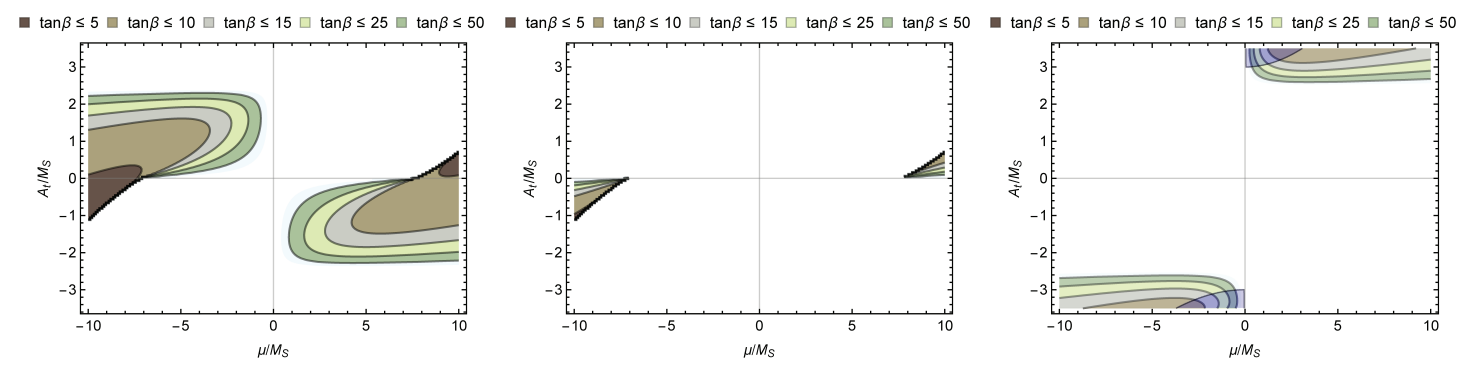

Figure 1: Contours of $\tan \beta$ corresponding to exact alignment, $Z_{6}=0$, in the $\left(\mu / M_{S}, A_{t} / M_{S}\right)$ plane. $Z_{1}$ is adjusted to give the correct Higgs mass. The leading one-loop and two-loop corrections of $O\left(\alpha_{s} h_{t}^{2}\right)$ to $Z_{1}$ and $Z_{6}$ have been included. Taking the three panels together, one can immediately discern the regions of zero, one, two and three values of $\tan \beta$ in which exact alignment is realized. Taken from Ref. [50].

In light of the SM-like nature of the observed Higgs boson, the ATLAS Collaboration concluded that $m_{A} \gtrsim 370 \mathrm{GeV}$ [51]. However, this analysis failed to consider the possibility of approximate alignment without decoupling [47], which can be achieved in certain regions of the MSSM parameter space [50]. The direct searches for $H$ and $A$ (decaying into $\tau^{+} \tau^{-}$) by the ATLAS and CMS Collaborations in the mass region from $200-370 \mathrm{GeV}$ provide no constraints for values of $\tan \beta \lesssim 8-10[52,53]$. In this parameter regime, approximate alignment is still possible for suitable choices of $\mu / M_{S}$ and $A_{t} / M_{S}$. A recent pMSSM parameter scan [50], which takes into account the observed Higgs data, direct searches for $H$ and $A$, indirect constraints from heavy flavor physics, and supersymmetric particle searches, finds that values of $m_{A}$ as low as $200 \mathrm{GeV}$ are within $2 \sigma$ of the best fit point obtained by a global likelihood analysis, under the assumption that $h$ is the observed Higgs boson of mass $125 \mathrm{GeV}^{5}$

Alignment without decoupling can also be achieved in the NMSSM (where an additional Higgs singlet superfield is added to the MSSM). For further details, see Ref. [54].

\subsection{Beyond Higgs singlets and doublets}

If one considers a scalar sector with triplet Higgs fields, then one must also include additional Higgs multiplets in such a way that the electroweak $\rho$-parameter is approximately equal to 1 . Georgi and Machacek constructed a model in which $\rho=1$ at tree-level due to a well chosen scalar potential that respects the custodial symmetry [55]. Their model contains a complex $Y=1$ doublet, a complex $Y=2$ triplet and a real $Y=0$ singlet. After minimizing the scalar potential, there is a doublet vev, $v_{\phi}$, and a common triplet vev, $v_{\chi}$, with $v^{2} \equiv v_{\phi}^{2}+8 v_{\chi}^{2} \simeq(246 \mathrm{GeV})^{2}$.

The physical scalars make up custodial SU(2) multiplets: a 5-plet of states $\left(H_{5}^{ \pm \pm}, H_{5}^{ \pm}\right.$and $\left.H_{5}^{0}\right)$ with common mass $m_{5}$, a triplet $\left(H_{3}^{ \pm}, H_{3}^{0}\right)$ with common mass $m_{3}$, and custodial singlets that mix with squared mass matrix [56]

$$
\mathscr{M}^{2}=\left(\begin{array}{cc}
Z_{11} v_{\phi}^{2} & v_{\phi} v_{\chi}\left(Z_{12}-2 \sqrt{3} m_{3}^{2} / v^{2}\right) \\
v_{\phi} v_{\chi}\left(Z_{12}-2 \sqrt{3} m_{3}^{2} / v^{2}\right) & \frac{3}{2} m_{3}^{2}-\frac{1}{2} m_{5}^{2}+v_{\chi}^{2}\left(Z_{22}-12 m_{3}^{2} / v^{2}\right)
\end{array}\right),
$$

where the $Z_{i j}$ depend on the dimensionless quartic couplings. The custodial singlet CP-even Higgs bosons are $h$ and $H$ with $m_{h} \leq m_{H}$. An approximate alignment limit can be realized in two dif-

\footnotetext{
${ }^{5}$ The same analysis also yields an allowed parameter regime where $H$ is the observed Higgs boson of mass $125 \mathrm{GeV}$.
} 
ferent ways. First, if $v_{\chi} \ll v$, then $h$ is SM-like if $Z_{11} v^{2}<\frac{3}{2} m_{3}^{2}-\frac{1}{2} m_{5}^{2}$; otherwise, $H$ is SM-like. Alternatively, in the decoupling limit, $h$ is SM-like and $m_{H} \simeq m_{3} \simeq m_{5} \gg m_{h}$ [56].

One interesting feature of the Georgi-Machacek model is that the existence of doubly-charged Higgs bosons modifies the unitarity sum rule [57],

$$
\sum_{i} g_{h_{i} W^{+} W^{-}}^{2}=g^{2} m_{W}^{2}+\sum_{k}\left|g_{H_{k}^{++} W^{-} W^{-}}\right|^{2}
$$

where the sum is taken over all CP-even Higgs bosons of the model. The presence on the last term on the right hand side of eq. (3.22) means that individual $h_{i} V V$ couplings can exceed the corresponding SM Higgs coupling to $V V$. It is convenient to write $c_{H} \equiv \cos \theta_{H}=v_{\phi} /\left(v_{\phi}^{2}+8 v_{\chi}^{2}\right)^{1 / 2}$, and $s_{H} \equiv \sin \theta_{H}$. Then, the following couplings are noteworthy [58]:

$$
\begin{aligned}
& H_{1}^{0} W^{+} W^{-}: \quad g c_{H} m_{W}, \quad H_{1}^{\prime 0} W^{+} W^{-}: \sqrt{8 / 3} g m_{W} s_{H}, \\
& H_{5}^{0} W^{+} W^{-}: \quad \sqrt{1 / 3} g m_{W} s_{H}, \quad H_{5}^{++} W^{-} W^{-}: \quad \sqrt{2} g m_{W} s_{H},
\end{aligned}
$$

where $H_{1}^{0}$ and $H_{1}^{\prime 0}$ are the custodial singlet interaction eigenstates. Among the four Higgs states that couple to $W W$, whose couplings are listed above, $H_{1}^{\prime 0}, H_{5}^{0}$ and $H_{5}^{++}$have no coupling to fermions, whereas the $H_{1}^{0} f \bar{f}$ coupling is $-g m_{q} /\left(2 m_{W} c_{H}\right)$.

In general $H_{1}^{0}$ and $H_{1}^{\prime 0}$ can mix. In the absence of $H_{1}^{0}-H_{1}^{\prime 0}$ mixing, $c_{H}=1$ corresponds to the alignment limit. But consider the strange case of $s_{H}=\sqrt{3 / 8}$, where the $H_{1}^{\prime 0}$ coupling to $W^{+} W^{-}$ matches that of the SM. Nevertheless, this does not saturate the $H W W$ sum rule! Moreover, it is possible that the $H_{1}^{\prime 0} W^{+} W^{-}$coupling is larger than the SM value of $g m_{W}$, without violating the sum rule given by eq. (3.22). Including $H_{1}^{0}-H_{1}^{\prime 0}$ mixing allows for even more baroque scenarios that are not possible in a multi-doublet extension of the SM Higgs sector.

\section{Conclusions}

Given the non-minimal nature of the observed spectrum of fundamental fermions and gauge bosons, it would be remarkable if the Higgs boson were a solo act. Thus, the search for additional scalars that exist in an extended Higgs sector will be an important enterprise in the experimental program at the LHC and at any future collider facility.

The current Higgs data strongly suggest that the observed Higgs boson is SM-like. This already places a strong constraint on the theoretical structure of any non-minimal Higgs sector. In particular, the alignment limit, in which the mass eigenstate corresponding to the observed Higgs boson is aligned with the direction (in field space) of the scalar doublet vev, must be a good approximation. The simplest way to achieve the alignment limit is in the case where all additional Higgs scalars are significantly heavier than the observed Higgs boson (corresponding to the decoupling limit). But, we have also argued for the possibility of the alignment limit without decoupling if the mixing between the SM Higgs boson and the additional neutral Higgs scalars is suppressed, in which case all Higgs scalars may be light [of $O(v)$ ] and thus more accessible to LHC searches.

Finally, as the Higgs data become more precise, deviations from SM properties of the Higgs boson may eventually be observed. Indeed, departures from the alignment limit encode critical information that can provide important clues for the structure of the non-minimal Higgs sector. Pursuing Higgs physics into the future by theorists and experimentalists is likely to lead to profound insights into the fundamental theory of particles and their interactions. 


\section{Acknowledgments}

This work is supported in part by the U.S. Department of Energy grant number DE-SC0010107. Travel support and the hospitality of Rikard Enberg, Arnaud Ferrari and Uppsala University are also gratefully acknowledged.

\section{References}

[1] G. Aad et al. [ATLAS Collaboration], Phys. Lett. B 716 (2012) 1.

[2] S. Chatrchyan et al. [CMS Collaboration], Phys. Lett. B 716 (2012) 30.

[3] See e.g., K. Nakamura and S.T. Petkov, Neutrino mass, mixing, and oscillations, in the 2016 Review of Particle Physics, C. Patrignani et al. [Particle Data Group], Chin. Phys. C 40 (2016) 100001.

[4] See e.g., M. Drees and G. Gerbier, Dark Matter, in the 2016 Review of Particle Physics, op. cit.

[5] See e.g., G.A. White, A Pedagogical Introduction to Electroweak Baryogenesis (IOP Publishing, Bristol, UK, 2016).

[6] See e.g., J.E. Kim and G. Carosi, Rev. Mod. Phys. 82 (2010) 557.

[7] See e.g., A. Hebecker and J. Hisano, Grand Unified Theories, in the 2016 Review of Particle Physics, op. cit.

[8] See e.g., J. Ellis and D. Wands, Inflation, in the 2016 Review of Particle Physics, op. cit.

[9] See e.g., M. J. Mortonso, D.H. Weinberg and M. White, Dark Energy, in the 2016 Review of Particle Physics, op. cit.

[10] V. F. Weisskopf, Phys. Rev. 56 (1939) 72.

[11] See e.g., H.E. Haber, Supersymmetry, Part I (Theory), in the 2016 Review of Particle Physics, op. cit.

[12] See e.g., C. Csaki, C. Grojean and J. Terning, Rev. Mod. Phys. 88 (2016) 045001.

[13] V. Agrawal, S. M. Barr, J.F. Donoghue and D. Seckel, Phys. Rev. Lett. 80 (1998) 1822; Phys. Rev. D 57 (1998) 5480.

[14] N. Arkani-Hamed, K. Blum, R.T. D’ Agnolo and J. Fan, JHEP 1301 (2013) 149.

[15] P. Draper, H. E. Haber and J. T. Ruderman, JHEP 1606 (2016) 124.

[16] G. Aad et al. [ATLAS and CMS Collaborations], JHEP 1608 (2016) 045.

[17] J.F. Gunion and H.E. Haber, Phys. Rev. D 67 (2003) 075019.

[18] N. Craig, J. Galloway and S. Thomas, arXiv:1305.2424 [hep-ph].

[19] See Chapter 1 of D.M. Asner et al., ILC Higgs White Paper, arXiv:1310.0763 [hep-ph].

[20] M. Carena, I. Low, N.R. Shah and C.E.M. Wagner, JHEP 1404 (2014) 015.

[21] H.E. Haber, in Proceedings of the of the Toyama International Workshop on Higgs as a Probe of New Physics 2013 (HPNP2013), arXiv:1401.0152 [hep-ph].

[22] H. Georgi and D. V. Nanopoulos, Phys. Lett. 82B (1979) 95.

[23] G.C. Branco, L. Lavoura and J.P. Silva, CP Violation (Oxford University Press, Oxford, UK, 1999).

[24] S. Davidson and H.E. Haber, Phys. Rev. D 72 (2005) 035004 [E: Phys. Rev. D 72 (2005) 099902]. 
[25] H.E. Haber and Y. Nir, Nucl. Phys. B 335 (1990) 363.

[26] P.S. Bhupal Dev and A. Pilaftsis, JHEP 1412 (2014) 024 [E: JHEP 1511 (2015) 147].

[27] A. Pilaftsis, Phys. Rev. D 93 (2016) 075012.

[28] R. Barbieri, L.J. Hall and V.S. Rychkov, Phys. Rev. D 74 (2006) 015007.

[29] V. Silveira and A. Zee, Phys. Lett. 161B (1985) 136.

[30] C.P. Burgess, M. Pospelov and T. ter Veldhuis, Nucl. Phys. B 619 (2001) 709.

[31] H. Davoudiasl, R. Kitano, T. Li and H. Murayama, Phys. Lett. B 609 (2005) 117.

[32] G.M. Pruna and T. Robens, Phys. Rev. D 88 (2013) 115012.

[33] T. Robens and T. Stefaniak, Eur. Phys. J. C 75 (2015) 104; Eur. Phys. J. C 76 (2016) 268.

[34] J.F. Gunion, H.E. Haber, G.L. Kane and S. Dawson, The Higgs Hunter's Guide (Westview Press, Boulder, CO, 2000).

[35] G.C. Branco, P.M. Ferreira, L. Lavoura, M.N. Rebelo, M. Sher and J.P. Silva, Phys. Rept. 516(2012) 1.

[36] H.E. Haber and D. O’Neil, Phys. Rev. D 74 (2006) 015018 [E: Phys. Rev. D 74 (2006) 059905].

[37] H.E. Haber and D. O’Neil, Phys. Rev. D 83 (2011) 055017.

[38] H.E. Haber and O. Stäl, Eur. Phys. J. C 75 (2015) 491 [E: Eur. Phys. J. C 76 (2016) 312].

[39] J. Bernon, J.F. Gunion, H.E. Haber, Y. Jiang and S. Kraml, Phys. Rev. D 92 (2015) 075004.

[40] J. Bernon, J.F. Gunion, H.E. Haber, Y. Jiang and S. Kraml, Phys. Rev. D 93 (2016) 035027.

[41] S.L. Glashow and S. Weinberg, Phys. Rev. D 15 (1977) 1958.

[42] E.A. Paschos, Phys. Rev. D 15 (1977) 1966.

[43] H.E. Haber, G.L. Kane and T. Sterling, Nucl. Phys. B 161 (1979) 493.

[44] L.J. Hall and M.B. Wise, Nucl. Phys. B 187 (1981) 397.

[45] J.F. Donoghue and L.F. Li, Phys. Rev. D 19 (1979) 945.

[46] See e.g., P. Draper and H. Rzehak, Phys. Rept. 619 (2016) 1.

[47] M. Carena, H.E. Haber, I. Low, N.R. Shah and C.E.M. Wagner, Phys. Rev. D 91 (2015) 035003.

[48] H.E. Haber, R. Hempfling and A.H. Hoang, Z. Phys. C 75 (1997) 539.

[49] M. Carena, H.E. Haber, S. Heinemeyer, W. Hollik, C.E.M. Wagner and G. Weiglein, Nucl. Phys. B 580 (2000) 29.

[50] P. Bechtle, H.E. Haber, S. Heinemeyer, O. Stäl, T. Stefaniak, G. Weiglein and L. Zeune, Eur. J. Phys. C, in press [arXiv:1608.00638 [hep-ph]].

[51] G. Aad et al. [ATLAS Collaboration], JHEP 1511 (2015) 206.

[52] The ATLAS Collaboration, ATLAS-CONF-2016-085.

[53] The CMS Collaboration, CMS-PAS-HIG-16-037.

[54] M. Carena, H.E. Haber, I. Low, N.R. Shah and C.E.M. Wagner, Phys. Rev. D 93 (2016) 035013.

[55] H. Georgi and M. Machacek, Nucl. Phys. B 262 (1985) 463.

[56] K. Hartling, K. Kumar and H.E. Logan, Phys. Rev. D 90 (2014) 015007.

[57] J.F. Gunion, H.E. Haber and J. Wudka, Phys. Rev. D 43 (1991) 904.

[58] J.F. Gunion, R. Vega and J. Wudka, Phys. Rev. D 42 (1990) 1673. 